\title{
Fabrication of High Thermal Conductivity NARloy-Z- Diamond Composite Combustion Chamber Liner for Advanced Rocket Engines
}

\author{
Biliyar N. Bhat \\ Materials and Processes Laboratory \\ NASA- Marshall Space Flight Center, Huntsville, Alabama \\ January 6, 2016
}

$57^{\text {th }}$ AIAA Structures, Structural Dynamics and Materials Conference

San Diego, CA 


\section{Materials and Processes Development Team}

- NASA-MSFC

- Biliyar Bhat - Principal Investigator

- Sandra Greene - Co-Investigator - chamber design and testing

- Enrique Jackson - thermal conductivity

- James Coston, Ellen Rabenberg - microscopy

- Will Tilson/Jacobs - tensile testing

- Supported by NASA-MSFC Technology Investment Project

- NASA-GRC

- Dr. David Ellis - consultant

- Penn State - Applied Research Laboratory

- Dr. Jogender Singh - FAST processing

- Momentive Performance Materials

- Aaron Rape - thermal conductivity

- Global Technology Enterprises

- Dr. Sion Pickard - coated diamonds 


\section{Overview}

- Introduction

- Improving thermal conductivity of copper alloys

- Project goals

- Chamber liner fabrication process

- Field Assisted Sintering Technology (FAST)

- Diffusion bonding

- Fabrication challenges

- Results

- Follow on work 


\section{Introduction}

- NARloy-Z alloy (Cu-3Ag-0.5Zr) is state of the art material for making combustion chamber liner for liquid rocket engines. Thermal conductivity (TC)- $320 \mathrm{~W} / \mathrm{mK}$

- Currently used in RS-25, RS-68

- Improved TC will help to improve the performance of rocket engines

- Improved turbopump power, thrust to weight ratio, specific impulse

- Prior work on NARloy-Z-Diamond composites showed promise

- $50 \%$ improvement in thermal conductivity relative to copper

- Technology development goals:

- Fabricate a subscale combustion chamber liner (TRL 4)

- Fabricate test chamber assembly

- Hot fire test to demonstrate performance improvements (TRL 5) 


\section{Thermal conductivity of Cu-Ag-Zr-D composites}

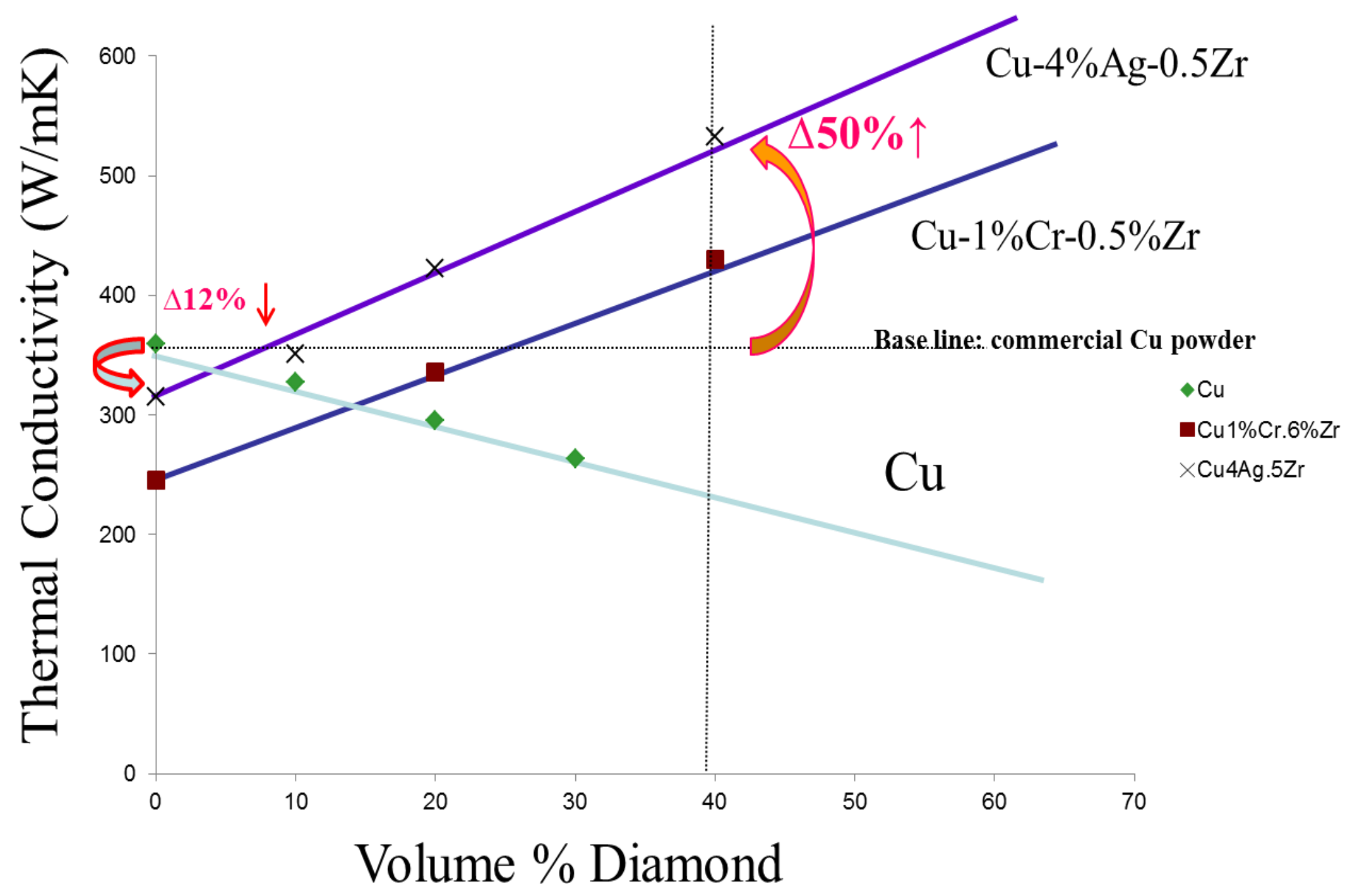




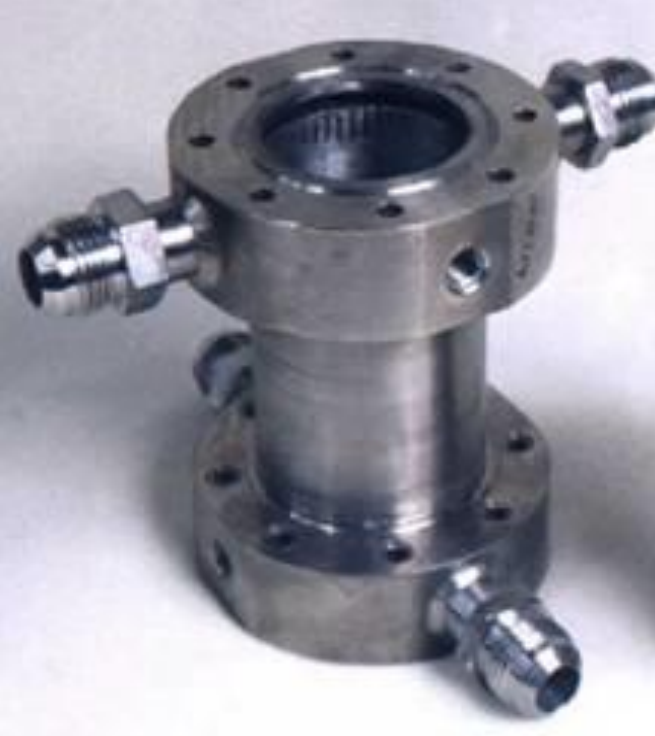

B

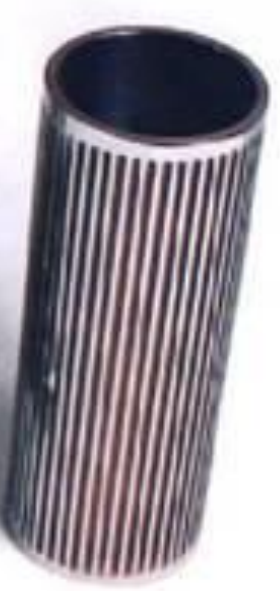

A

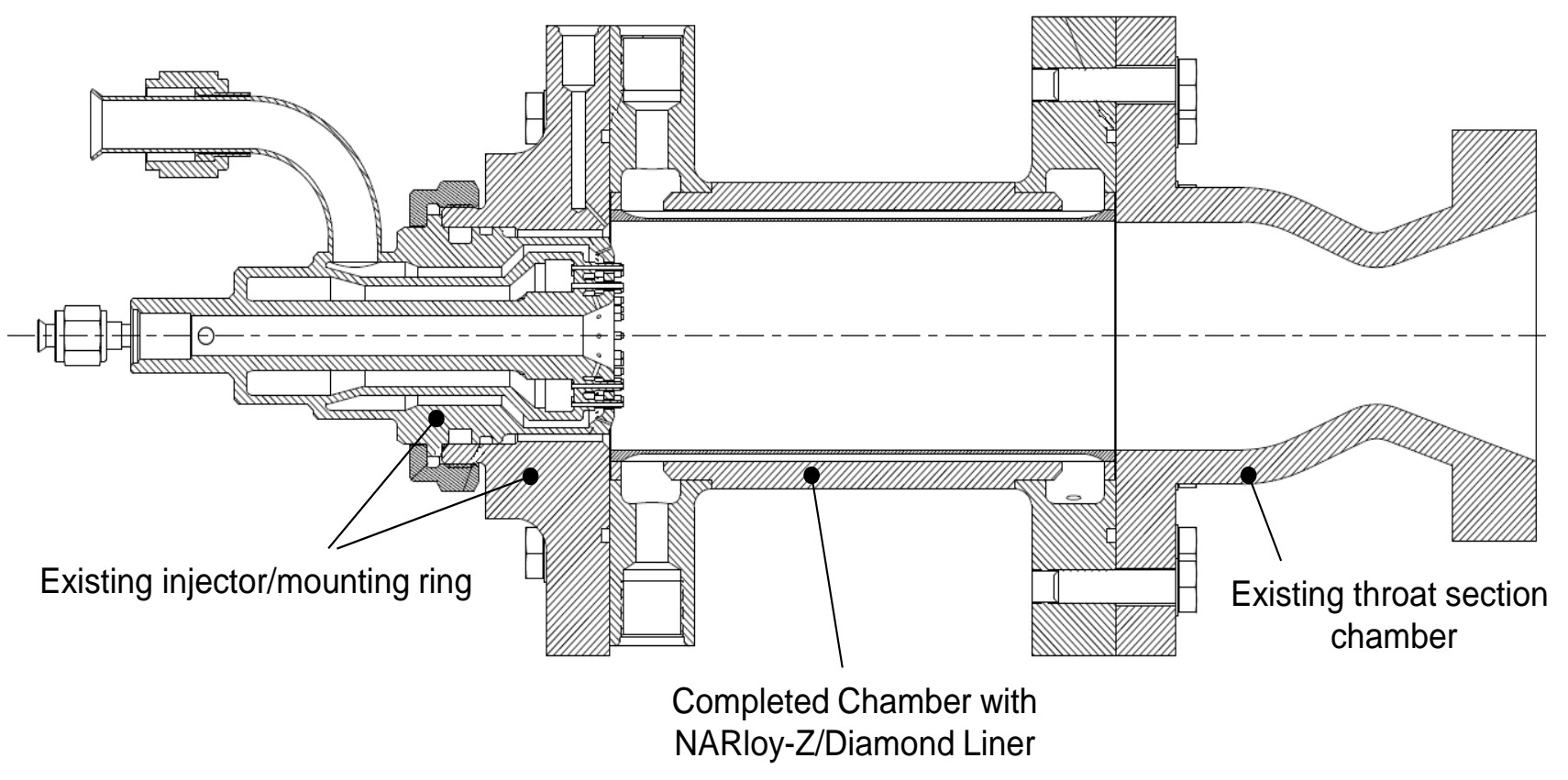

- Chamber liner (A) - 2.75"OD, 2.5" ID, 8" long

Hot fire test assembly schematic

- Chamber assembly (B) 


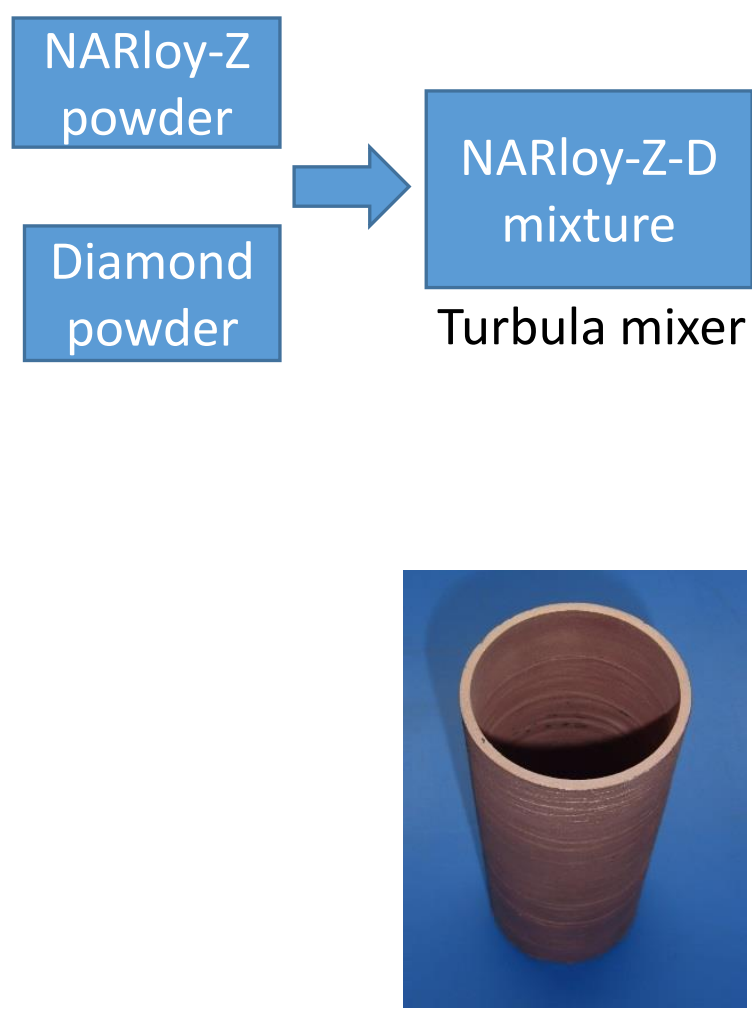

Finished liner
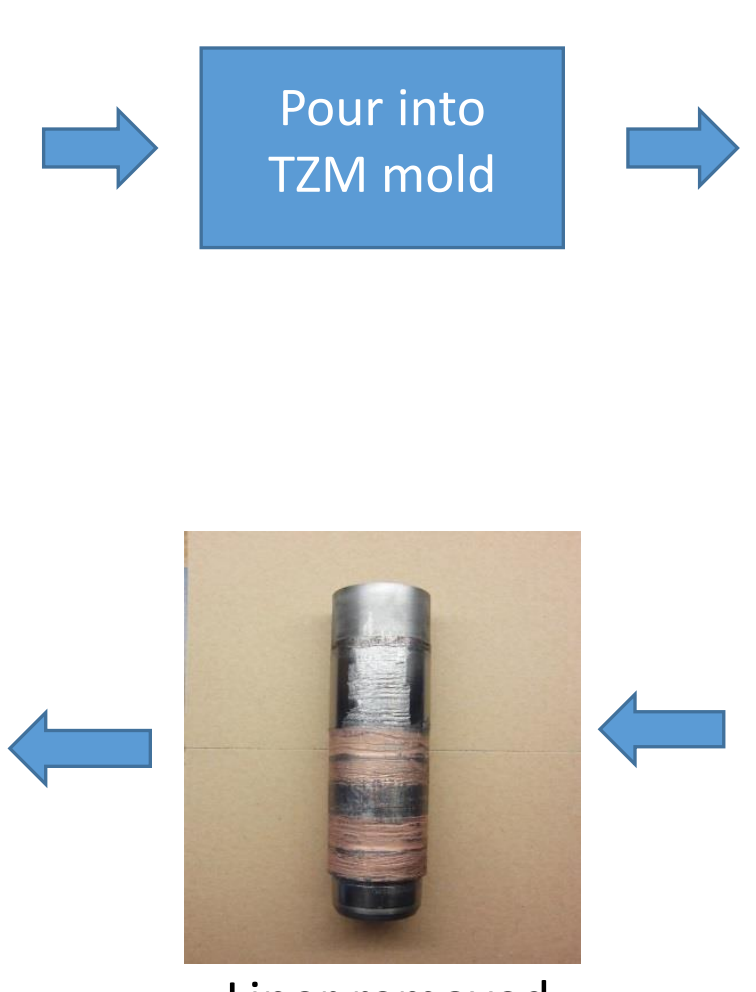

Liner removed from mold
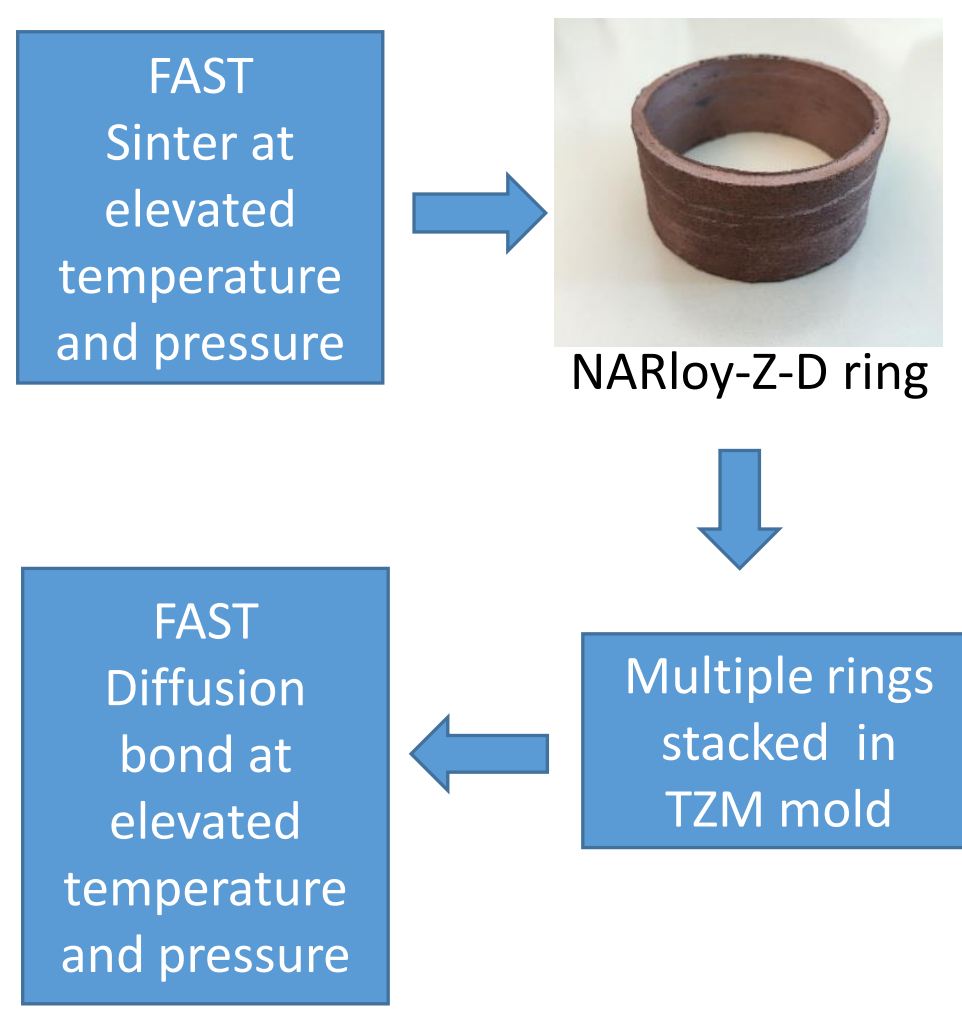

Multiple rings stacked in TZM mold 


\section{Field Assisted Sintering Technology (FAST)}

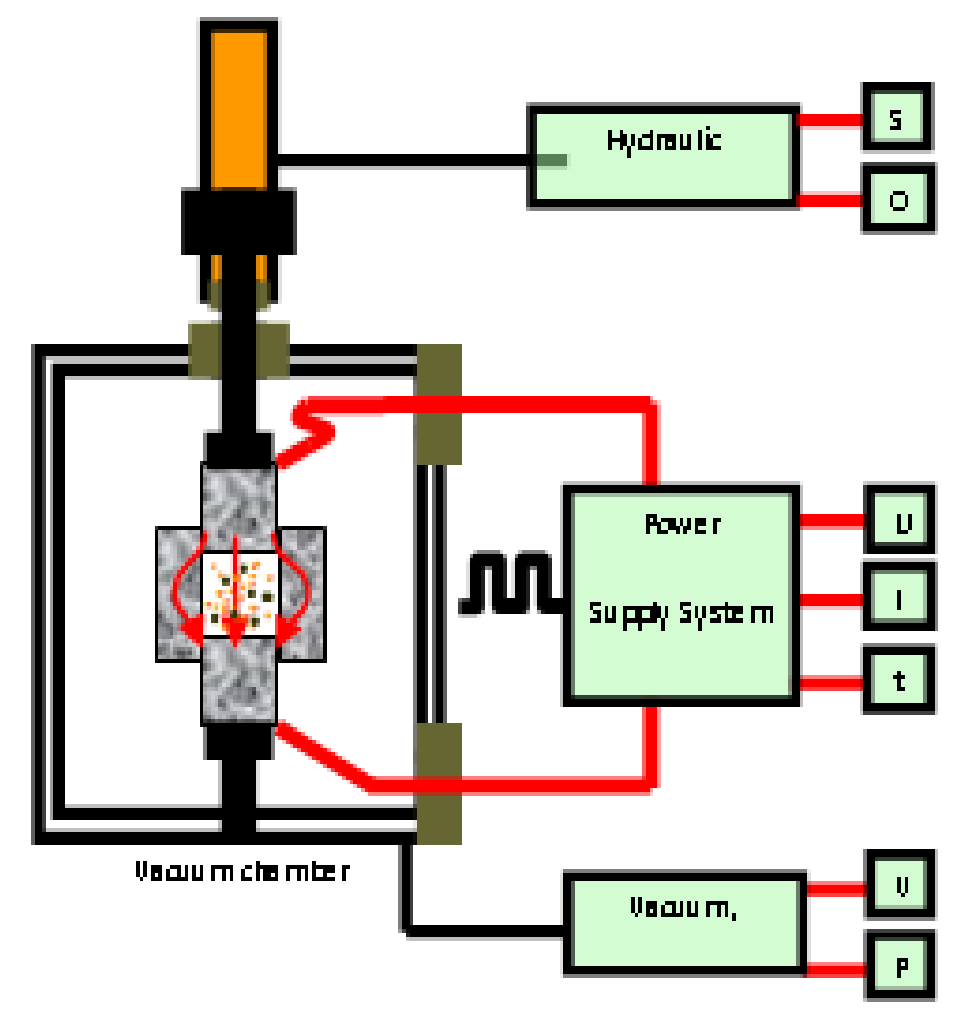

FAST - schematic

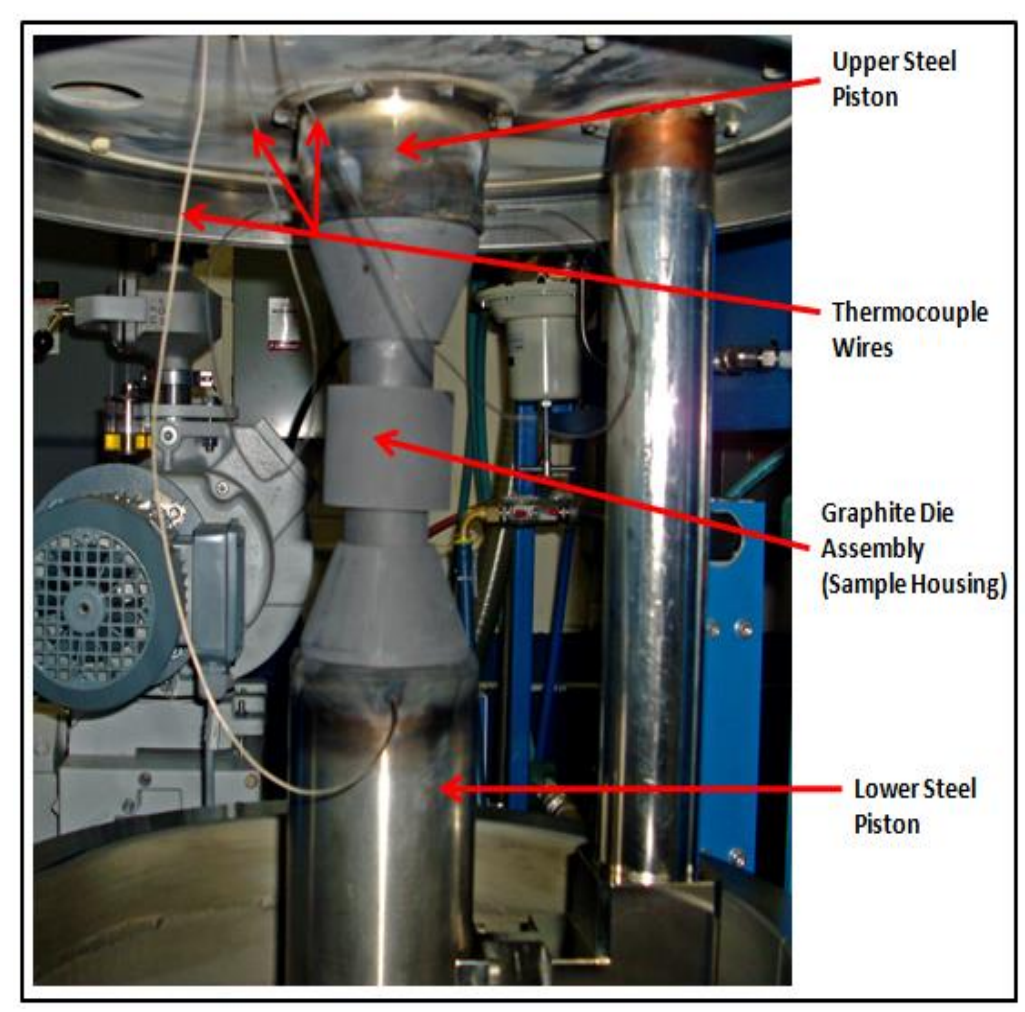

FAST system at Penn State - ARL

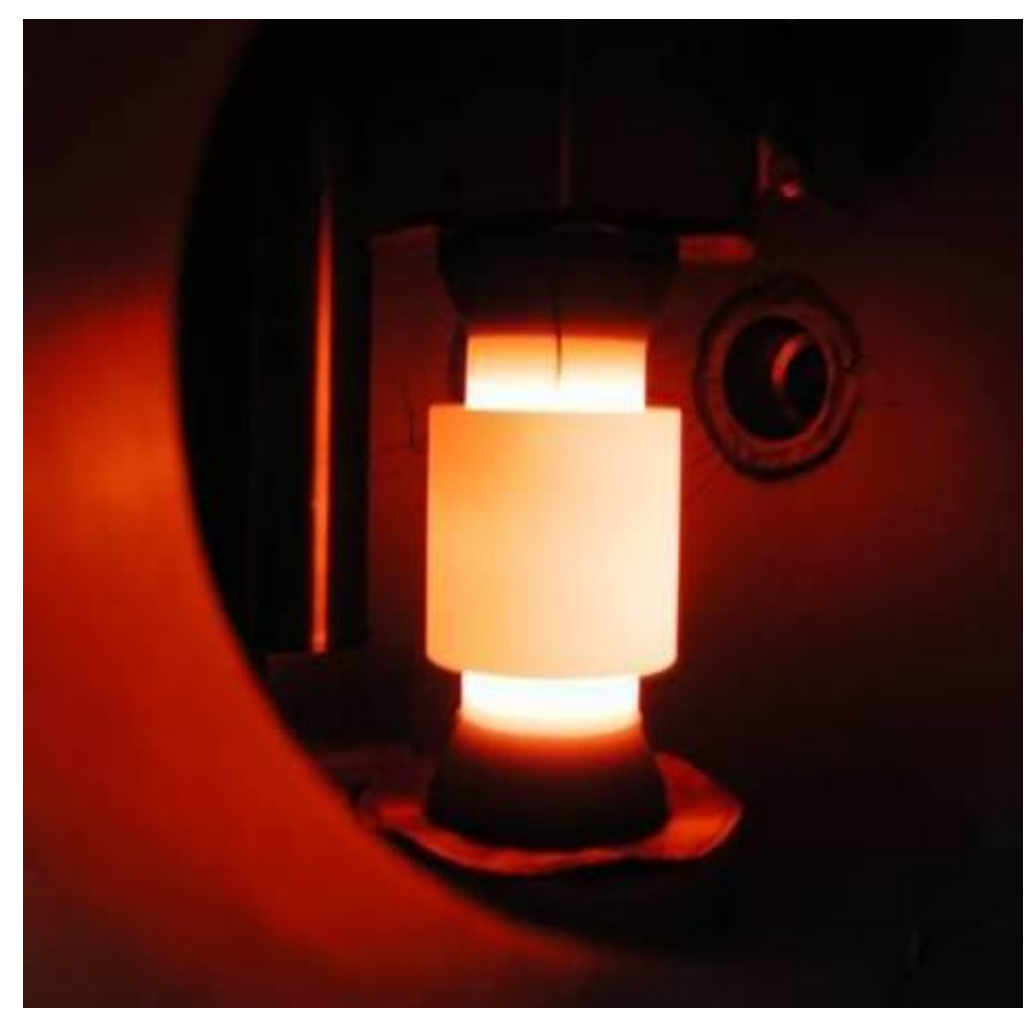

Sintering at high temperature in FAST apparatus 


\section{Diffusion bonding (schematic)}

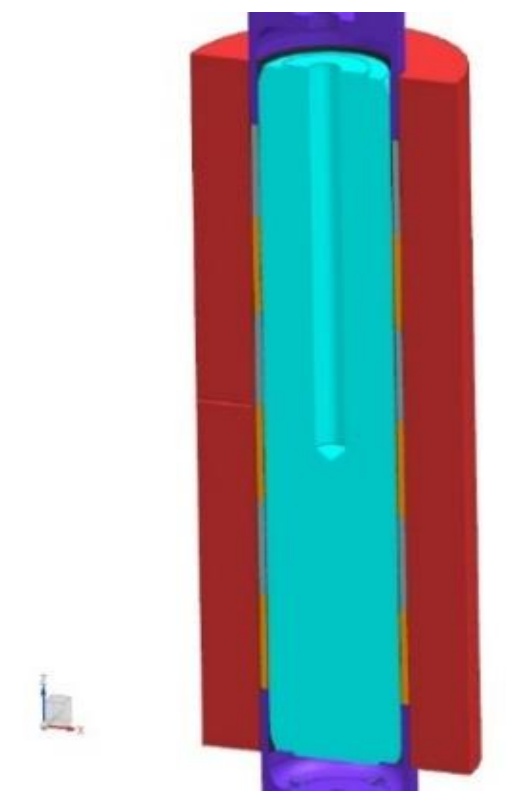

Eight rings stacked inside TZM mold for joining
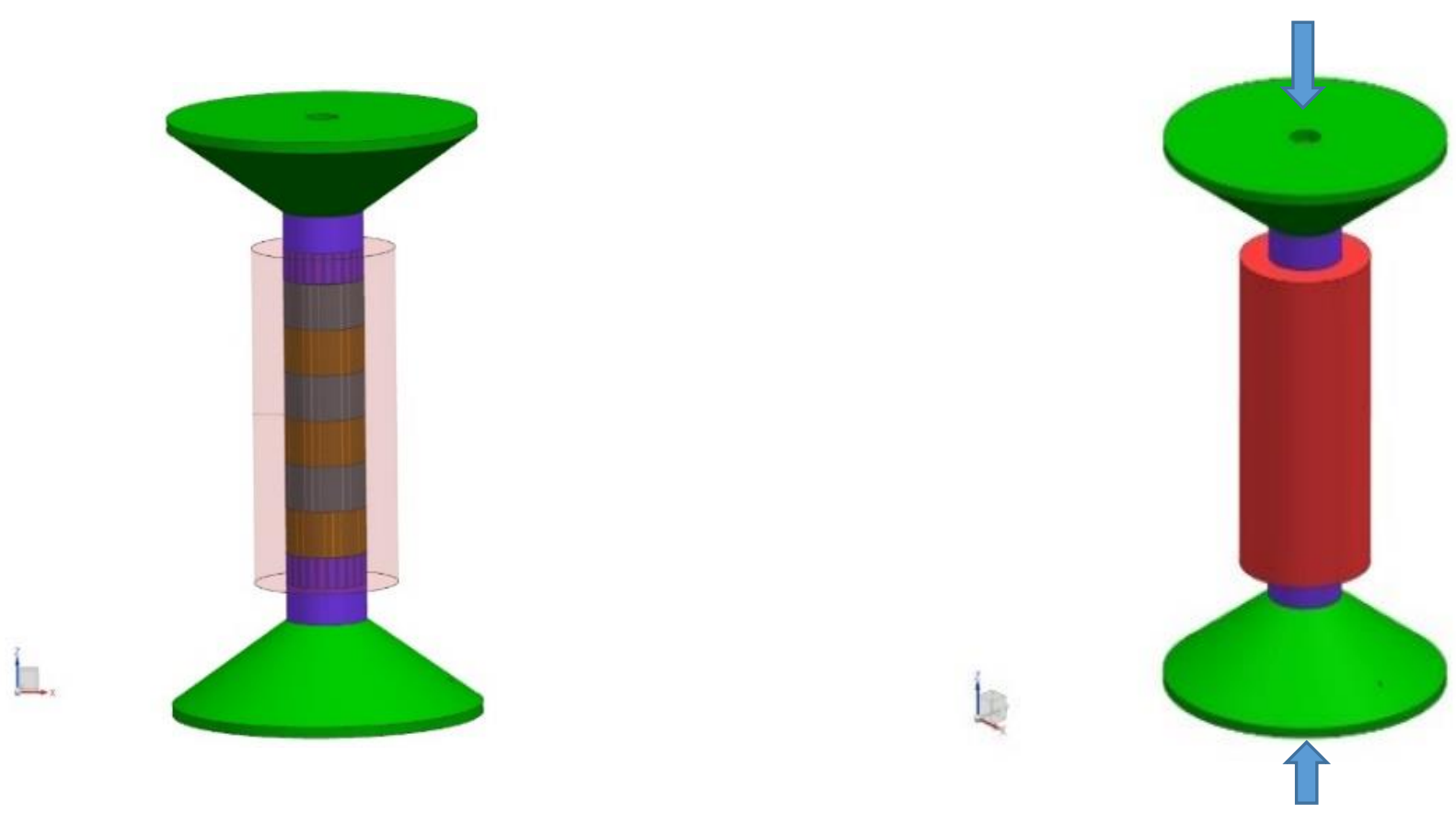

Translucent model with multicolors showing the rings
Diffusion bonding by FAST 


\section{Fabrication challenges}

- Machining of NARloy-Z-D composite

- Too hard to machine by conventional means

- EDM and water jet cutting successful

- Near net shape forming

- Switching from graphite to TZM molds for better strength at elevated temperatures

- Segregation of diamonds in microstructure

- Diamonds segregate easily - hard to homogenize

- Metal coatings help to improve mixing - Ti, $\mathrm{Cu}$

- Cu coating worked better

- Copper coated diamonds - supplied by GTE

- Coating of MoC for better contact conductance

- Overcoat of Cu for better mixing and sintering

- Diffusion bonding of NARloy-D rings

- Interlayer of NARloy-Z for better bonding

- Microscopy and NDE

- Material is too hard to make metallographic samples

- Freshly fractured surfaces the best way to examine microstructure in SEM

- CT scanning to assess segregation and ensure quality 


\section{Tensile properties (preliminary)}

\begin{tabular}{|c|c|c|c|c|c|}
\hline Sample type & Composition & \begin{tabular}{|c|} 
Test \\
temperature, \\
Environment
\end{tabular} & YS, ksi & UTS, ksi & Elongation, \% \\
\hline NARloy-Z & Base line & $75^{\circ} \mathrm{F}$, air & 18 & 45 & 33 \\
\hline NARloy-Z-30D & 30 vol\% diamond & $75^{\circ} \mathrm{F}$, air & 19 & 19 & $<1$ \\
\hline NARloy-Z-40D & 40 vol\% diamond & $75^{\circ} \mathrm{F}$, air & $18-20$ & $18-24$ & $<1$ \\
\hline NARloy-Z-40D & 40 vol\% diamonds & $935^{\circ} \mathrm{F}, \mathrm{GN} 2$ & 11 & 11 & $<1$ \\
\hline NARloy-Z-30(Ti-D) & 30 vol\% Ti-coated diamond & $75^{\circ} \mathrm{F}$, air & 12 & $12-13$ & $<1$ \\
\hline NARloy-Z-30 (Cu-MoC-D) & $\begin{array}{l}28 \text { vol\% diamonds, Cu-MoC } \\
\text { coated }\end{array}$ & $70^{\circ} \mathrm{F}$, air & 18 & 23 & $2-3$ \\
\hline NARloy-Z-30 (Cu-MoC-D) & $\begin{array}{l}28 \text { vol\% diamonds, Cu-MoC } \\
\text { coated }\end{array}$ & $\begin{array}{l}1000^{\circ} \mathrm{F}, 250 \\
\text { psi He }\end{array}$ & $5-6$ & $5-7$ & $2-3$ \\
\hline $\begin{array}{l}\text { Diffusion bonded } \\
\text { NARloy-Z-40D }\end{array}$ & $\begin{array}{l}40 \text { vol.\% Diamond; NARloy-Z } \\
\text { at bond line }\end{array}$ & $70^{\circ} \mathrm{F}$, air & 10 & 11 & $<1$ \\
\hline
\end{tabular}




\section{Thermal conductivity (preliminary)}

\begin{tabular}{|l|c|c|l|}
\hline $\begin{array}{c}\text { Sample chemistry } \\
\text { (vol\%) }\end{array}$ & $\begin{array}{c}\text { Thermal conductivity } \\
(\mathrm{W} / \mathrm{m}-\mathrm{K})\end{array}$ & $\begin{array}{c}\text { Temperature, } \\
{ }^{0} \mathrm{~K}\end{array}$ & \multicolumn{1}{|c|}{\begin{tabular}{l} 
Comments \\
\hline NARloy-Z
\end{tabular}} \\
\hline NARloy-Z-30\%D & 320 & 380 & $\begin{array}{l}\text { Base line (Ref. 2) } \\
\text { Diamond segregation } \\
\text { observed (Ref. 3) }\end{array}$ \\
\hline NARloy-Z-40\%D & 344 & 380 & $\begin{array}{l}\text { Diamond segregation } \\
\text { observed (Ref. 3) }\end{array}$ \\
\hline NARloy-30\%TiD & 176 & 300 & Ti lowers TC \\
\hline NARloy-Z-28\%CuD & 462 & 300 & TC acceptable \\
\hline
\end{tabular}




\section{Microstructure}

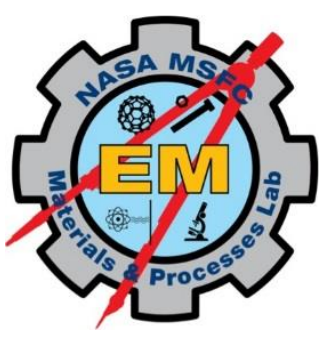

30 percent vf_c01_25x

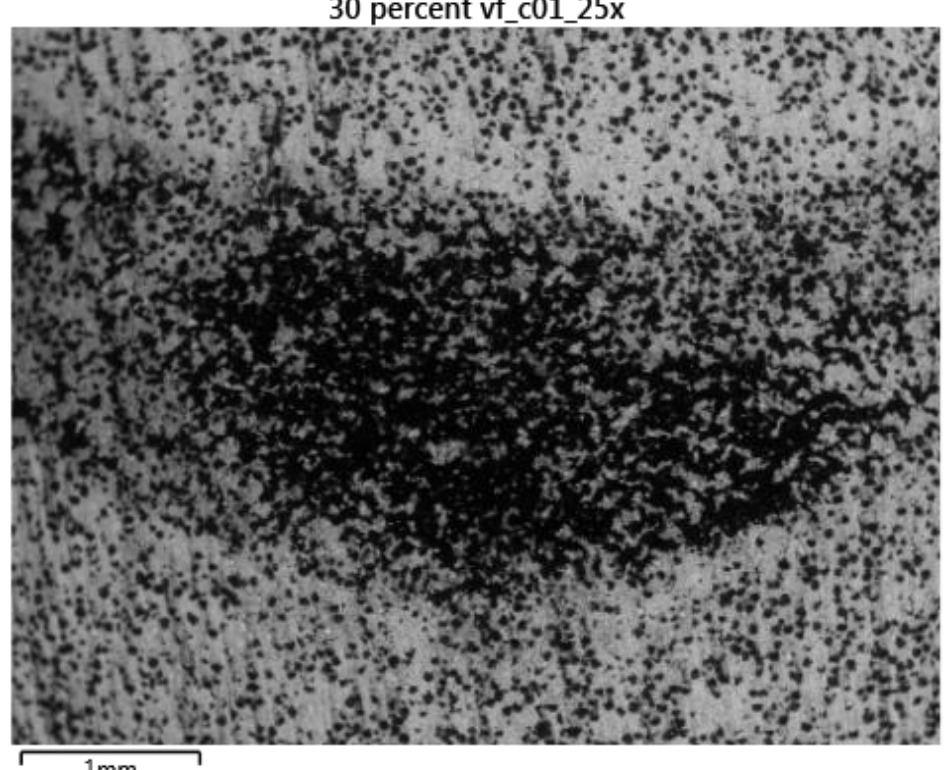

SEM Micrograph showing diamond segregation (dark area) in NARloy-Z-30\%D Composite

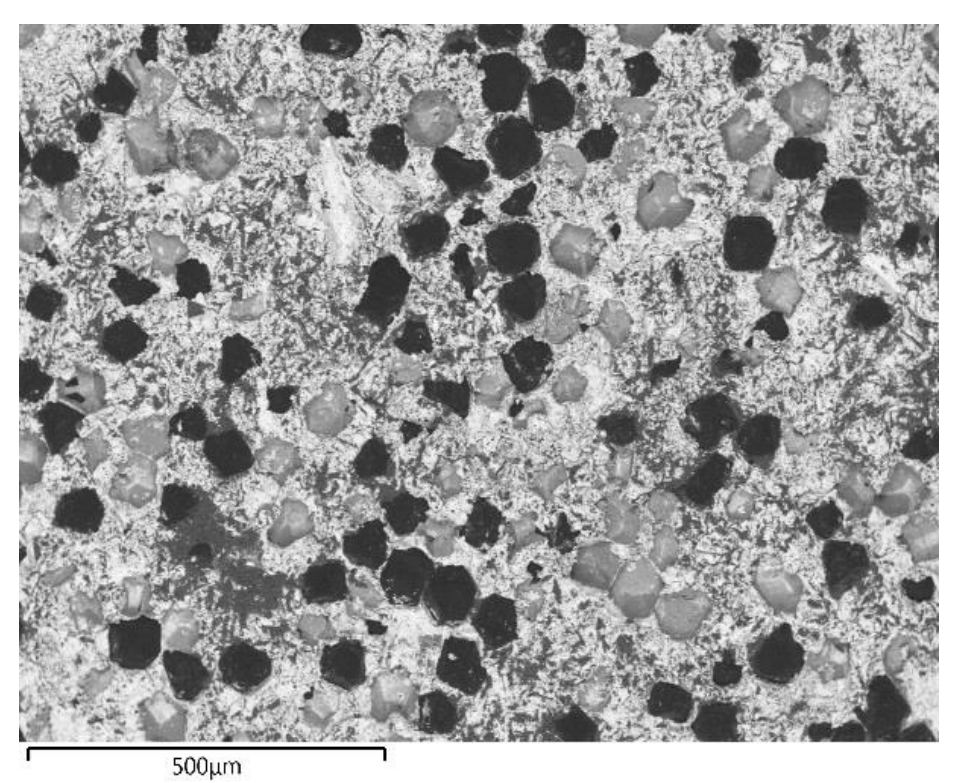

SEM fractograph of NARloy-ZTi coated diamond composite

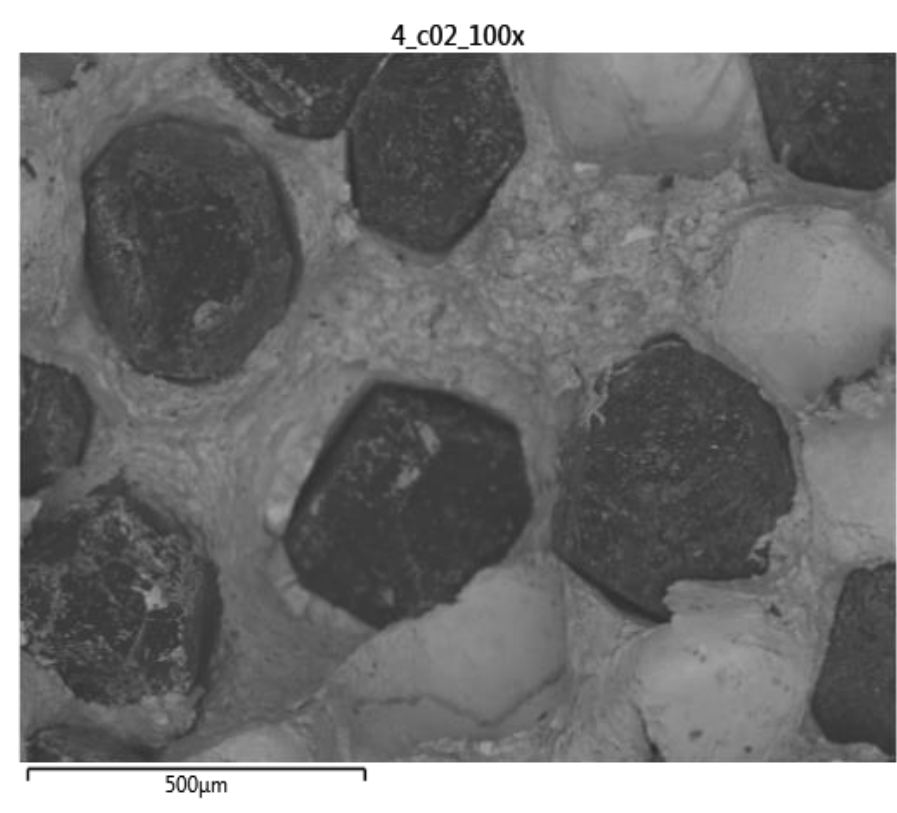

SEM fractograph of NARloy-Z-CuMoC coated diamond composite 


\section{Chamber liner}

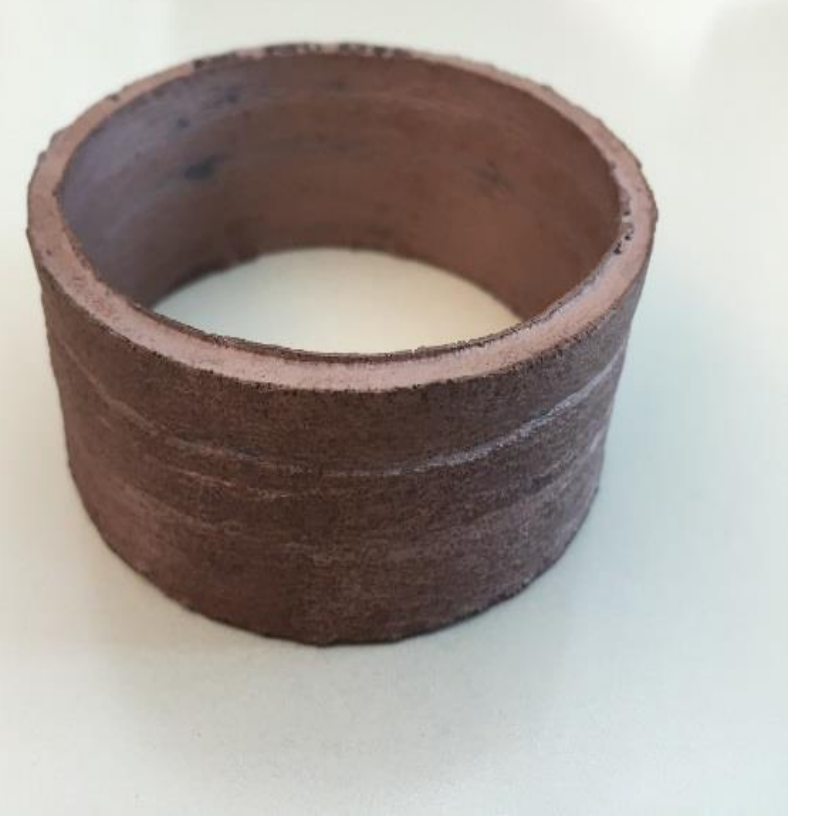

Chamber liner ring (2.5" ID., 2.75" OD, 1.0" long) made from NARloy-Z- CuD composite

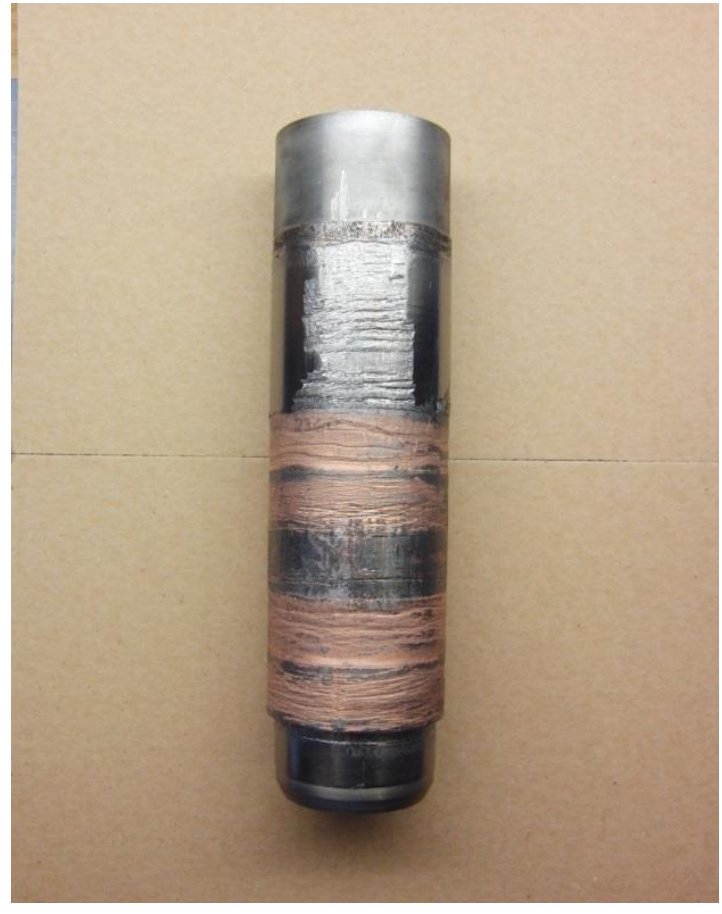

NARloy-Z chamber liner fabricated by FAST - after taking out of the mold

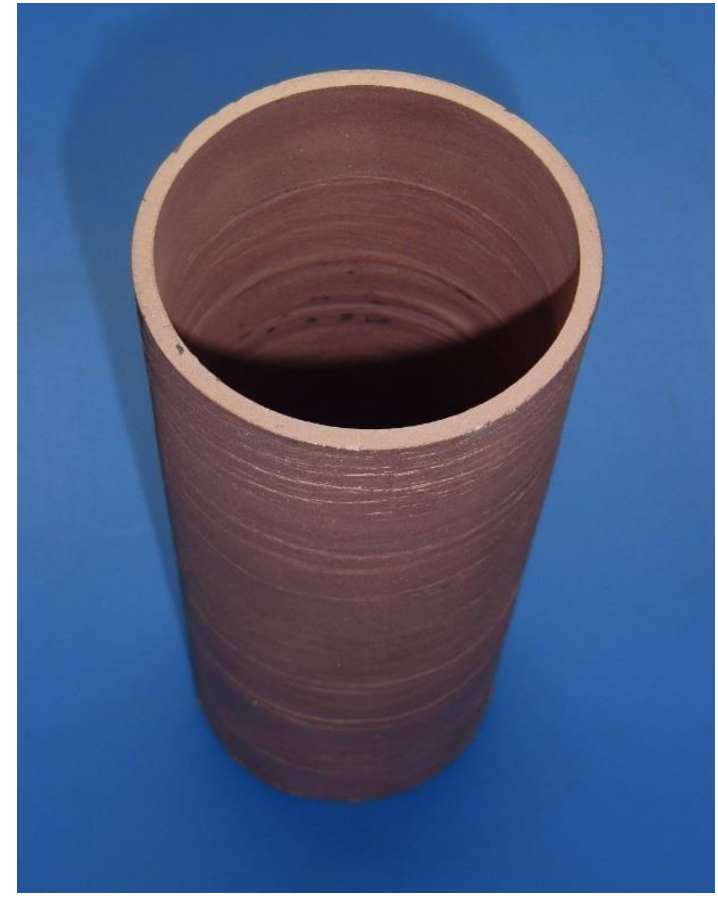

NARloy-Z chamber liner after cleaning 


\section{Follow on work}

- Diffusion bond NARloy-Z-CuD composite rings in FAST apparatus

- Machine cooling channels by water jet grinding

- Electroplate with nickel to close out the channels

- Fabricate coolant manifolds and integrate with hot fire test assembly

- Hot fire test in MSFC test stand 115

- Analyze data and assess performance

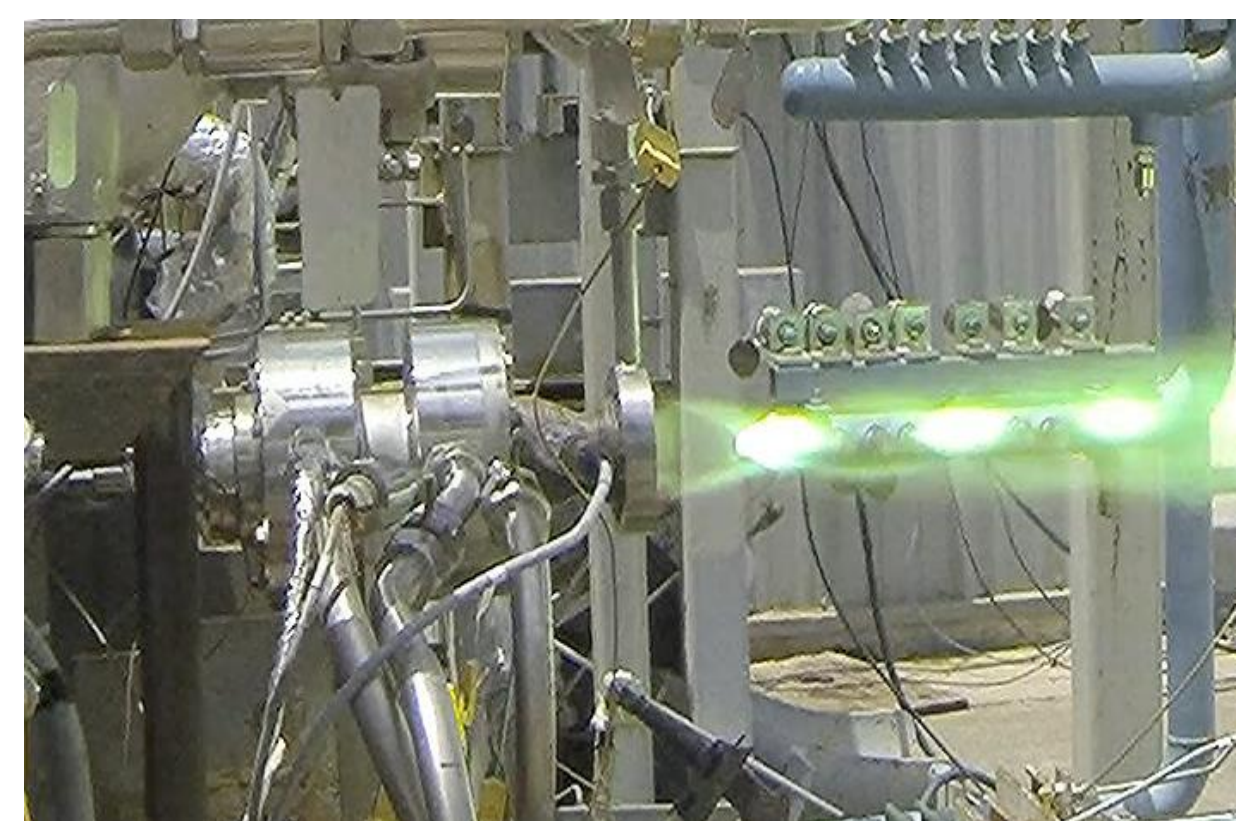

Hot fire testing at MSFC TS 115 


\section{Summary and Conclusions}

- Successfully formulated a high thermal conductivity NARloy-Z-CuD composite material that can be processed into shapes.

- Developed processing technique for combustion chamber liner rings by use of Field Assisted Sintering Technology (FAST)

- Developed fabrication technique for chamber liner by diffusion bonding

- This is a break through technology in metal matrix composites, which will help to make our future propulsion systems lighter and higher performance using a high thermal conductivity material for combustion chamber liner.

- Materials and processing technologies can be developed further to optimize properties for specific applications, e.g., heat exchangers and other thermal management systems. 